OPEN@ACCESS

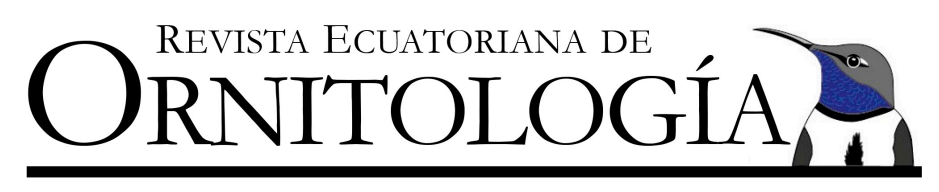

COMUNICACIÓN CORTA/SHORT COMMUNICATION

\title{
Depredación del Tucán Andino Piquilaminado Andigena laminirotris (Ramphastidae) a una serpiente del género Chironius (Colubridae)
}

\author{
William A. Arteaga-Chávez*, Dayana P. Togán-Murillo \\ Universidad Central del Ecuador, Facultad de Ciencias Biológicas, Carrera de Biología. Quito, Ecuador. \\ *Autor para correspondencia/Corresponding author, e-mail: arteagawilliam95@yahoo.es
}

Editado por/Edited by: Boris A. Tinoco

Recibido/Received: 25 Marzo 2019 Aceptado/Accepted: 29 Enero 2020

Publicado en línea/Published online: 22 Marzo 2020

\section{Plate-billed Mountain Toucan Andigena laminirostris (Ramphastidae) depredation on a Chironius snake (Colubridae)}

\begin{abstract}
Resumen
El Tucán Andino Piquiliaminado Andigena laminirostris es una especie principalmente frugívora que complementa su dieta con invertebrados, vertebrados pequeños, huevos y pichones de otras aves. En esta nota reportamos el primer evento de depredación de A. laminirostris a una serpiente del género Chironius (Colubridae). Este reporte presenta aspectos ecológicos anteriormente desconocidos de A. laminirostris.
\end{abstract}

Palabras clave: Andigena laminirostris, Chironius, Colombia, depredación, dieta.

\begin{abstract}
Plate-billed Mountain-Toucan Andigena laminirostris is primarily frugivorous, but complements its diet with invertebrates, small vertebrates, eggs and nestlings of other birds. In this note, we present the first record of A. laminirostris preying upon a snake of the genus Chironius (Colubridae). This note presents behavioral data previously unknown for A. laminirostris.
\end{abstract}

Keywords: Andigena laminirostris, Chironius, Colombia, depredation, diet.

Existen cuatro especies de tucanes andinos del género Andigena, todas confinadas al norte de los Andes (Remsen et al., 2019), con un patrón de reemplazo geográfico o altitudinal entre especies (Fjeldså \& Krabbe, 1990; Schulenberg et al., 2010; Ayerbe-Quiñones, 2018; Freile \& Restall, 2018). La distribución geográfica de dos de estas especies de tucanes andinos se restringe a dos Áreas de Endemismo de Aves: el Tucán Andino Encapuchado A. cucullata, en las partes superiores de Yungas, en el sureste de Perú y noroeste de Bolivia, y el Tucán Andino Piquilaminado A. laminirostris en el Chocó, en el suroeste de Colombia y noroeste de Ecuador (Fjeldså \& Krabbe, 1990; Stattersfield et al., 1998; BirdLife International, 2019).

Andigena laminirostris se considera como Vulnerable en Ecuador (Freile et al., 2019), En Peligro en Colombia (Renjifo et al., 2014) y Casi amenazado globalmente (BirdLife International, 2019), debido principalmente a la fragmentación y pérdida de hábitats. Habita bosques nublados subtropicales, primarios y secundarios maduros, entre 1600-2600 m s.n.m., donde se mueve principalmente en parejas por el subdosel (Ridgely \& Greenfield, 2006). Aunque es localmente común, de tamaño relativamente grande, plumaje conspicuo y bastante vocal, se ha documentado poco sobre su historia natural (Renjifo et al., 2014). 
Su dieta es primordialmente frugívora (Beltrán, 1994; Short \& Sharpe, 2019). Cumple un rol ecológico importante como dispersor de semillas, debido a que se mueve largas distancias entre parches de bosque (Holbrook, 2011). Además, se han documentado pocas observaciones anecdóticas de depredación de vertebrados (Beltrán, 1994; Solano-Ugalde, 2011; V. Portillo com. pers., 2019). En esta nota presentamos el primer registro de depredación de una serpiente por parte de A. laminirostris.

El 14 de Octubre de 2017, mientras observábamos aves en el sendero El Mirador-Las Piñas (1,16108, -77,97539, 1850 m s.n.m.), en la Reserva Natural La Planada, departamento de Nariño, vertiente occidental de los Andes de Colombia, observamos a un individuo de A. laminirostris capturar y alimentarse de una serpiente. A las $c$. $11 \mathrm{~h} 00$ este individuo se movía rápidamente de una rama a otra en el dosel, a unos $10 \mathrm{~m}$ del suelo. De pronto, realizó un ataque directo y atrapó algo con su pico. Al momento de la observación pudimos determinar que se trataba de un probable colúbrido por la forma de la cabeza, forma del cuerpo, coloración verde oscura y cola delgada y larga (Torres-Carvajal et al., 2019). El tucán sostuvo a la culebra de forma transversal con su pico, y la golpeó contra el tronco. La serpiente se envolvía en el cuello y pico del tucán. El tucán la dejó caer, pero la capturó nuevamente unos $3 \mathrm{~m}$ antes de que llegara al suelo (Fig. 1A).

Cautelosamente nos acercamos hasta $c .4 \mathrm{~m}$ para observar mayores detalles. Determinamos que la culebra medía aproximadamente 1,20 m de longitud. El tucán no huyó ante nuestra presencia, y tampoco abandonó a su presa. La sostenía firmemente con el pico, y la sacudió nuevamente. Usando sus patas alternadamente, la presionó contra el tronco y la mantuvo inmóvil. Continuó picoteando fuertemente hasta matarla. Los picotazos se enfocaron principalmente en la sección del cuerpo en donde se encuentran los principales órganos (e.g., pulmones y corazón), no en la cabeza. Empezó a rasgar y sacar trozos pequeños de carne con su pico, sujetando a la culebra con una pata. Los órganos fueron las primeras partes que ingirió. Después arrancó la cabeza, parte del cuello yparte del cuerpo, y los tragó (Figs. 1B y 1C). Por último, intentó tragarse la mitad restante de la culebra, pero no lo consiguió y la dejó caer. Todo este evento de depredación duró aproximadamente 30 min. El colúbrido fue identificado posteriormente, a partir de los registros fotográficos, como una especie indeterminada del género Chironius (Colubridae).

Al igual que todas las especies de Ramphastidae, A. laminirostris se considera principalmente frugívora (Remsen et al., 1993). Sin embargo, Beltrán (1994) y Short \& Sharpe (2019) incluyen en la dieta de esta especie a insectos, caracoles, huevos, pichones y roedores. Solano-Ugalde (2011) documentó a una pareja de $A$. laminirostris alimentándose de un ilulo Caecilia sp. (Amphibia, Caecilidae) en la Reserva Bellavista, provincia de Pichincha, Ecuador. En esta misma localidad, el 3 de marzo de 2019, Juan Carlos Figueroa (com. pers., 2019) observó a una pareja de A. laminirostris alimentarse de una lombriz gigante en el suelo. Además, en la Reserva El Bosque, departamento de Nariño, Colombia, Verónica Portillo (com. pers., 2019) observó un A. laminirostris sujetando con sus patas a un colúbrido y dándole picotazos. Sin embargo, el tucán voló con el colúbrido en su pico, por lo que no se pudo determinar si fue un evento completo de depredación.

Se ha documentado que durante el cuidado parental, A. laminirostris alimenta a sus crías con insectos (Coleoptera), caracoles (Isomeria sp. y Drymaeus sp.), huevos, polluelos de otras aves y micromamíferos, que constituyen cerca del 20\% de la dieta de origen animal (Beltrán, 1994). De igual manera, se han encontrado lombrices entre los alimentos entregados a un nido de un Tucán Andino Piquinegro A. nigrirostris (SolanoUgalde, 2011). Muchas aves mayormente frugívoras incluyen vertebrados en las dietas de sus crías por el aporte de proteínas para su desarrollo (Morton, 1973), pero los eventos de depredación por parte de individuos adultos son escasos.

Las serpientes del género Chironus son generalmente diurnas, terrestres y arbóreas, y se encuentran en diversos pisos altitudinales (Torres-Carvajal et al., 2019). Estas culebras se han documentado en la dieta de aves rapaces diurnas (e.g., Gavilán Coliblanco Geranoaetus albicaudatus y Halcón Reidor Herpetotheres cachinnans; Motta-Junior et al., 2010; Costa et al., 2014) y nocturnas (e.g., Búho Terrestre Athene cunicularia; Vieira \& Teixeira, 2008), sin reportes previos de depredación por tucanes.

El presente reporte constituye el primer registro de depredación de A. laminirostris a un colúbrido, y podría tratarse del ítem alimenticio más grande registrado hasta el momento para la especie y el género Andigena en general. Aunque esta observación puede representar un comportamiento alimenticio accidental, la estrategia de caza y la precisión para matar al colúbrido sugieren que A. laminirostris podría incluir en su dieta a colúbridos de manera oportunista. 

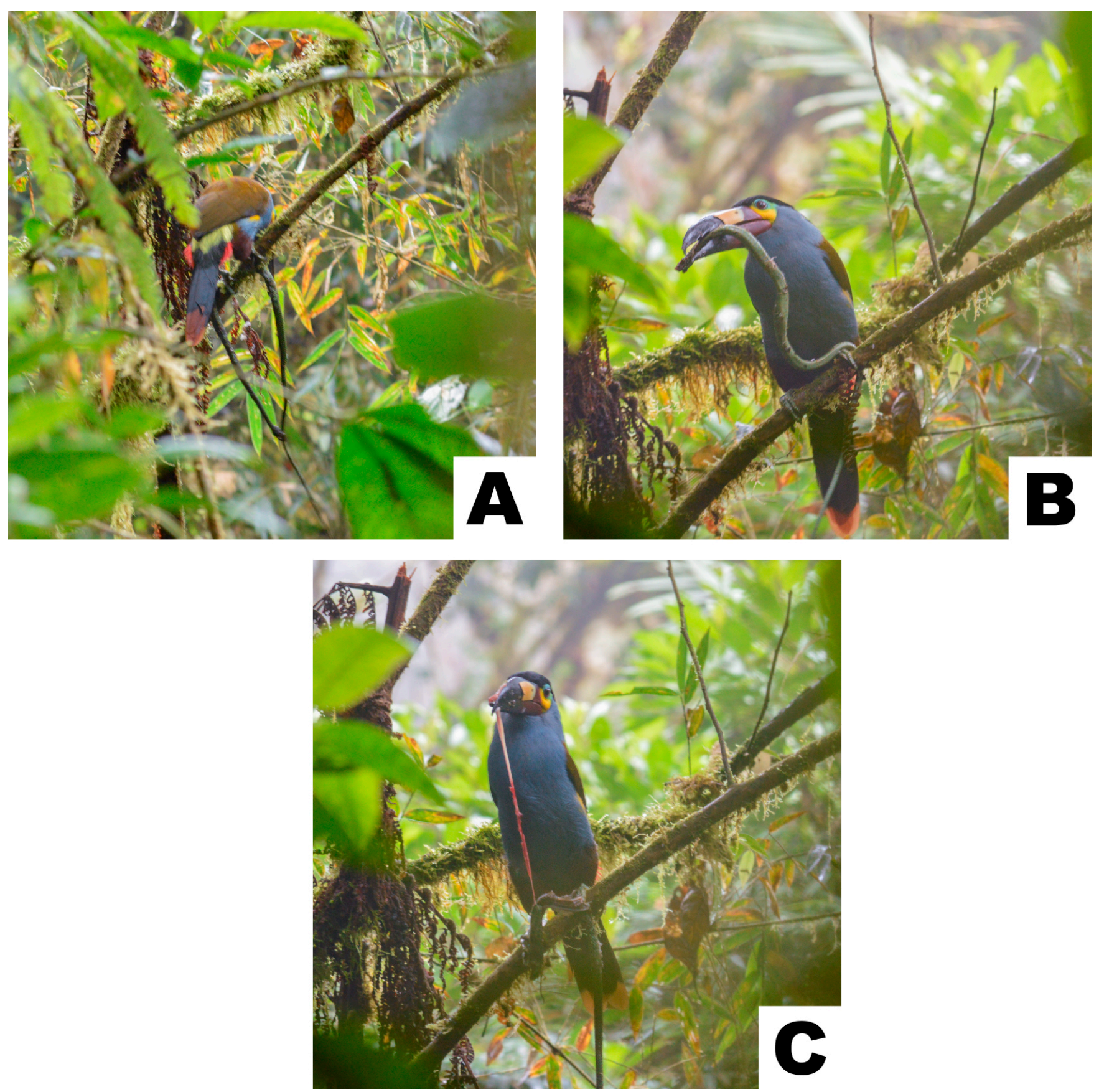

Figura 1: Tucán Andino Piquilaminado Andigena laminirostris alimentándose de una culebra del género Chironius (Colubridae), en la Reserva Natural La Planada, Nariño, Colombia. A) culebra recapturada después de haber intentado escapar. B) tucán sujeta el resto del cuerpo después de haberse comido los órganos, la cabeza y el cuello. C) tucán desgarra con el pico y come el resto del cuerpo (William A. Arteaga-Chávez y Dayana P. Togán-Murillo).

\section{AGRADECIMIENTOS}

A la Rerserva Natural La Planada por permitirnos el ingreso y uso de sus instalaciones, y a la Fundación Altrópico por el apoyo logístico. A Jorge H. Valencia por su ayuda en la identificación de la serpiente. A Jack Eitniear y Harold Greeney por compartir información especializada. Verónica Portillo y Juan Carlos Figueroa amablemente compartieron sus observaciones. Juan Freile y dos revisores anónimos revisaron y proporcionaron comentarios importantes que ayudaron a mejorar este manusrito. 


\section{REFERENCIAS}

Ayerbe-Quiñones, F. (2018). Guía ilustrada de la avifauna colombiana. Bogotá, Colombia: Wildlife Conservation Society.

Beltrán, J. W. (1994). Natural history of the Plate-billed Mountain Toucan Andigena laminirostris in Colombia. Center for the Study of Tropical Birds Miscellaneous Publications, 2, 1-91.

BirdLife International. (2019, March 7). Species factsheet: Andigena laminirostris. BirdLife International. URL: http://www.birdlife.org

Costa, H. C., Lopes, L. E., de Freitas, M. B., \& Zorzin, G. (2014). The reptile hunter's menu: a review of the prey species of Laughing Falcons, Herpetotheres cachinnans (Aves: Falconiformes). North-Western Journal of Zoology, 10(2), 445-453. URL: http://biozoojournals.ro/nwjz/content/v10n2.html

Freile, J. F., \& Restall, R. (2018). Birds of Ecuador. Londres, U.K.: Helm Field Guides.

Freile, J. F., Santander, T., Carrasco, L., Cisneros-Heredia, D. F., Guevara, E. A., Sánchez-Nivicela, M., \& Tinoco, B. A. (2019). Lista roja de las aves del Ecuador continental. Quito, Ecuador: Ministerio del Ambiente, Aves y Conservación, Comité Ecuatoriano de Registros Ornitológicos, Universidad del Azuay, Red Aves Ecuador y Universidad San Francisco de Quito.

Fjeldså, J., \& Krabbe, N. (1990). Birds of the high Andes. Copenhagen, Dinamarca: Apollo Books y University of Copenhagen.

Holbrook, K. M. (2011). Home range and movement patterns of toucans: implications for seed dispersal. Biotropica, 43(3), 357-364. DOI: https://doi.org/10.1111/j.1744-7429.2010.00710.x

Morton, E. S. (1973). On the evolutionary advantages and disadvantages of fruit eating in tropical birds. American Naturalist, 107(953), 8-22. DOI: https://doi.org/10.1086/282813

Motta-Junior, J. C., Granzinolli, M. A., \& Monteiro, A. R. (2010). Miscellaneous ecological notes on Brazilian birds of prey and owls. Biota Neotropica, 10(4), 255-259. DOI: http://dx.doi.org/10.1590/S1676$\underline{06032010000400042}$

Remsen, J. V., Hyde, M. A., \& Chapman, A. (1993). The diets of Neotropical trogons, motmots, barbets and toucans. Condor, 95(1), 178-192. DOI: https://doi.org/10.2307/1369399

Remsen, J. V., Areta, J. I., Cadena, C. D., Claramunt, S., Jaramillo, A., Pacheco, J. F., Robbins, M. B., Stiles, F. G., Stotz, D. F. \& Zimmer, K. J. (2019, March 5). A classification of the bird species of South America. American Ornithologists' Union. URL: www.museum.lsu.edu/ Remsen/SACCBaseline.htm

Renjifo, L. M., Gómez, M. F., Velásquez-Tibatá, J., Amaya-Villarreal, A. M., Kattan, G. H., Amaya-Espinel, J. D., \& Burbano-Girón, J. (2014). Libro rojo de aves de Colombia, volumen I: bosques húmedos de los Andes y la costa Pacífica. Bogotá, Colombia: Editorial Pontificia Universidad Javeriana e Instituto Alexander von Humboldt.

Ridgely, R. S., \& Greenfield, P. J. (2006). Aves del Ecuador: guía de campo. Quito, Ecuador: Academia de Ciencias Naturales de Filadelfia y Fundación de Conservación Jocotoco.

Schulenberg, T. S., Stotz, D. F., Lane, D. F., O’Neill, J. P., \& Parker, T. A. (2010). Birds of Peru. Revised edition. Princeton, NJ: Princeton University Press.

Short, L. L., \& Sharpe, C. J. (2019, March 7). Plate-billed Mountain-toucan (Andigena laminirostris). In: J. del Hoyo, A. Elliott, J. Sargatal, D.A. Christie, \& E. de Juana (Eds.), Handbook of the birds of the world alive. Barcelona, España: Lynx Edicions. URL: https://www.hbw.com/node/56084 
Solano-Ugalde, A. (2011). The Plate-billed Mountain Toucan (Andigena laminirostris) feeding on a Caecilia spp. (Gymnophiona: Amphibia). Boletín de la Sociedad Antioqueña de Ornitología, 20(2), 43-45. URL: http://www.sao.org.co/boletinsao.html

Stattersfield, A. J., Crosby, M. J., Long, A. J., \& Wege, D. C. (1998). Endemic Bird Areas of the world. Priorities for biodiversity conservation. Cambridge, U.K.: BirdLife Conservation Series 7.

Torres-Carvajal, O., Pazmiño-Otamendi, G., \& Salazar-Valenzuela, D. (2019, Febrero 04). Reptiles del Ecuador. Version 2019.0. Quito, Ecuador: Museo de Zoología, Pontificia Universidad Católica del Ecuador. URL: https://bioweb.bio/faunaweb/reptiliaweb

Vieira, L. A., \& Teixeira, R. L. (2008). Diet of Athene cunicularia (Molina, 1782) from a sandy coastal plain in southeast Brazil.Boletim do Museu de Biologia Mello Leitão,23(5), 5-14. URL: http://boletim.sambio.org.br/pdf/23 01.pdf 